\title{
Metabolic syndrome in patients attending psychiatric day centres: prevalence and associations
}

\author{
Anna Gubbins, ${ }^{1}$ John Lally, ${ }^{1,2}$ Colm McDonald ${ }^{1,2}$
}

The Psychiatrist (2012), 36, 326-331, doi: 10.1192/pb.bp.111.037374

${ }^{1}$ National University of Ireland, Galway, Ireland; ${ }^{2}$ West Galway Mental Health Services, Health Service Executive West, Galway, Ireland

Correspondence to John Lally (john.lally@nuigalway.ie)

First received 8 Oct 2011, final revision 21 Dec 2011, accepted 29 Feb 2012

\begin{abstract}
Aims and method To ascertain the prevalence of metabolic syndrome in individuals with chronic enduring mental illness attending community psychiatric day centres, and to identify the prevalence of individual cardiometabolic risk factors and the rate of screening for these abnormalities. One hundred participants with chronic enduring mental illness were recruited and investigated using physical assessments and comprehensive laboratory testing.
\end{abstract}

Results Of the 100 participants recruited, 55\% met the criteria for metabolic syndrome. There were similar prevalence rates between those with psychotic and non-psychotic disorders. Forty-four per cent of the patients had not been screened for metabolic parameters in the previous 12 months, and $43 \%$ of these met criteria for metabolic syndrome.

Clinical implications Chronic enduring mental illness is associated with high prevalence rates of metabolic syndrome regardless of diagnosis or use of antipsychotic medication. A substantial proportion of this standard cohort of patients attending day centres carry cardiovascular risk factors that remain unidentified, but should be screened for and intensively managed.
Individuals with severe mental illnesses such as schizophrenia or bipolar disorder have a reduced life expectancy compared with the general population, especially related to cardiovascular disease.$^{1-3}$ Furthermore, this gap has increased in recent years, especially with regard to schizophrenia ${ }^{3,4}$ and bipolar affective disorder. ${ }^{4}$ The standardised mortality ratio for death within 1 year of psychiatric in-patient care for people with schizophrenia increased from 1.6 in people discharged in 1999 to 2.2 in those discharged in 2006, and for people with bipolar disorder it increased from 1.3 in 1999 to 1.9 in $2006 .{ }^{5}$ The aetiology of this excess cardiovascular disease is multifactorial and most likely includes genetic and lifestyle factors as well as disease-specific and treatment effects. ${ }^{6}$

People with severe mental illness are likely to have a number of modifiable cardiovascular risk factors. The metabolic syndrome is a constellation of cardiovascular risk factors that helps to identify individuals at risk of cardiovascular disease and type 2 diabetes. It comprises a combination of physical and metabolic abnormalities which can be identified by abnormal values for: central obesity, hypertension, high-density lipoprotein (HDL) cholesterol, triglycerides, and fasting plasma glucose. ${ }^{7}$ These abnormal clinical and metabolic findings act to increase the risk of cardiovascular disease. It has been consistently shown that there is an increased prevalence of metabolic syndrome in people with psychotic illness, with one large study reporting that $52 \%$ of individuals in a population of psychiatric out-patients met the US National Cholesterol
Education Program-Adult Treatment Panel III (NCEP ATP III) criteria for metabolic syndrome. ${ }^{8}$ In the Clinical Antipsychotic Trials of Intervention Effectiveness (CATIE) study, over $30 \%$ of patients met the NCEP ATP III criteria for metabolic syndrome at baseline. ${ }^{9}$

Weight gain is a well-established side-effect of antipsychotic medication, in particular certain secondgeneration antipsychotic medications. The cumulative incidence of patients becoming overweight on clozapine exceeds $50 \%$, with a mean weight gain of $4.45 \mathrm{~kg}$ at 10 weeks of treatment, ${ }^{10}$ and a mean weight gain of $4.15 \mathrm{~kg}$ for olanzapine and $2.1 \mathrm{~kg}$ for risperidone. ${ }^{11}$ There is an increased risk of dyslipidaemia, insulin resistance, hyperglycaemia and new-onset type 2 diabetes, and this risk is particularly associated with the use of olanzapine and clozapine. ${ }^{12-16}$

The need for screening and monitoring cardiovascular risk factors in psychiatric populations is well documented, however, the evaluation of screening practices by clinicians has been shown to be suboptimal. ${ }^{15,16}$ In this study, we sought to ascertain the prevalence and associations of metabolic syndrome in a typical cohort of individuals with chronic enduring mental illness attending the psychiatric day centres within a catchment area mental health service. We wished to evaluate whether there was an increased prevalence of metabolic syndrome in individuals on antipsychotic medication, on high-dose antipsychotics and on antipsychotic polypharmacy. We also sought to assess the prevalence of the individual metabolic risk factors for 
cardiovascular disease in our study population and the extent to which they were being screened for.

\section{Method}

Participants who lived in the community and who were attending one of the three psychiatric day centres in the West Galway adult mental health services were recruited over a 4-week period in July 2011. Two of the day centres were located in an urban setting and the third was in a rural setting. All of the 136 individuals who were attending any of the three centres over that period were asked to participate. Completed data were obtained from 100 patients (73.5\%) and included in the study; 36 individuals were not included in the study, as complete anthropomorphic data could not be obtained from them.

Diagnoses were made in accordance with ICD-10 diagnostic criteria from clinical interviews and review of the medical notes. ${ }^{17}$ Clinical and demographic data including age, gender and ethnicity were collected from each individual. Treatment with high-dose antipsychotic medication was defined as receiving more than $100 \%$ of the British National Formulary maximum dose per day. ${ }^{18}$ For patients on more than one medication, this was calculated by converting their prescribed dose to its percentage of the maximum recommended dose for each drug and then summing the percentages. ${ }^{19}$

The International Diabetes Federation (IDF) definition of metabolic syndrome ${ }^{7}$ (Table 1) was used. In addition, glycated haemoglobin (HbAlc) $>6 \%$ was used to indicate impaired fasting glucose and was deemed to meet the criteria for metabolic syndrome. ${ }^{19}$ This was applied to individuals who had blood drawn by their general practitioners (GPs) in the previous 12 months and who were screened with an HbAlc level rather than with a fasting plasma glucose level $(n=16)$.

The medical records (both hospital and primary care) of these individuals were examined for evidence of measurement of fasting glucose, HbAlc, triglyceride or HDL cholesterol levels. Those who did not have these parameters assessed in the previous 12 months had morning blood samples drawn after a period of fasting of at least $12 \mathrm{~h}$ and tested for: fasting plasma glucose, HDL cholesterol, triglycerides, total cholesterol, low-density lipoprotein (LDL) cholesterol and HbAlc.

Although anthropomorphic measurements such as body mass index (BMI) could be derived from some of the patients' notes, this information was often insufficient for the purposes of diagnosing metabolic syndrome (e.g. few patients had a documented waist circumference). Therefore, all patients were assessed for anthropomorphic measurements for the present study. Height, weight and waist circumference were all measured using standardised techniques. The waist circumference was measured at the midpoint between the lower rib and the iliac crest with the patient standing. Standardised techniques were used to measure blood pressure. A medical history was obtained from each individual and included items such as psychiatric history, medical history (including hypertension, diabetes mellitus and dyslipidaemia), medication, family history (including ischaemic heart disease), smoking and exercise habits.

\section{Statistical analysis}

Statistical analysis was performed using the Statistical Package for Social Sciences (SPSS) 18.0 for Windows. The student $t$-test for parametric data and the $\chi^{2}$-test for non-parametric data were employed. All statistical tests were two-sided and the $\alpha$-level for statistical significance was 0.05 .

\section{Results}

The mean age of the participants was 58 years $($ s.d. $=12.3)$ and $55 \%$ of the study population were female. Most patients had a diagnosis of schizophrenia (50\%), recurrent depressive disorder (31\%) and bipolar affective disorder (9\%). Overall, $85 \%$ of participants were treated with antipsychotic medication, with $79 \%$ of patients taking atypical antipsychotics, most frequently olanzapine (34\%), quetiapine (23\%) and clozapine (9\%). In total, $63 \%$ of patients were treated with atypical antipsychotic monotherapy and $6 \%$ were treated with typical antipsychotic

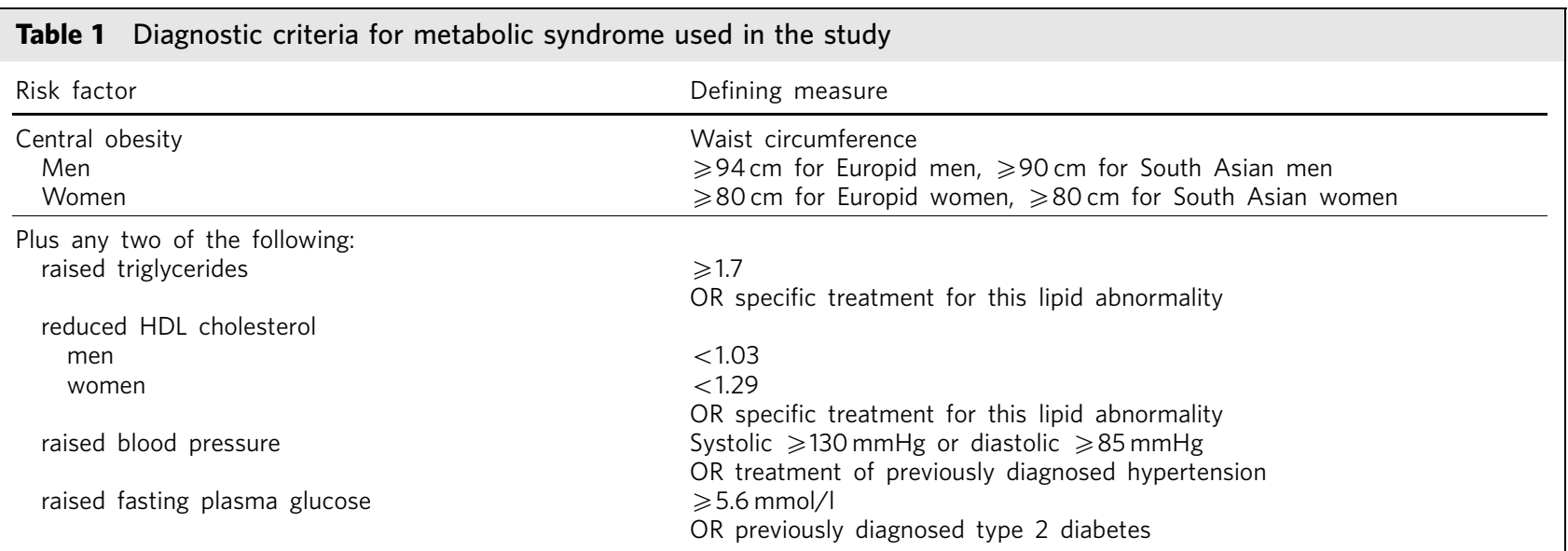


Table 2 Demographics and clinical characteristics of individuals with and without metabolic syndrome

\begin{tabular}{|c|c|c|c|c|c|}
\hline & $\begin{array}{l}\text { Metabolic syndrome } \\
\quad(n=55 ; 55 \%)\end{array}$ & $\begin{array}{l}\text { No metabolic syndrome } \\
\qquad(n=45 ; 45 \%)\end{array}$ & $\chi^{2}$-test & d.f. & $P$ \\
\hline Age, years: mean & $\begin{array}{c}59.7 \\
\text { (s.d. }=12.4 ; \text { range } 26-90)\end{array}$ & $\begin{array}{c}56.8 \\
(\text { s.d. }=12.2 ; \text { range } 34-87)\end{array}$ & $1.163^{a}$ & 98 & $0.255^{a}$ \\
\hline Male, $n(\%)$ & $20(45.5)$ & $24(54.6)$ & & & \\
\hline Female, $n(\%)$ & $35(62.5)$ & $21(37.5)$ & 2.893 & 1 & 0.089 \\
\hline \multicolumn{6}{|l|}{ Diagnosis, $n(\%)$} \\
\hline Schizophrenia & $26(52)$ & $24(48)$ & 0.364 & 1 & 0.564 \\
\hline Depression & $18(58.1)$ & $13(41.9)$ & 0.170 & 1 & 0.680 \\
\hline Bipolar disorder & $5(55.6)$ & $4(44.4)$ & 0.01 & 1 & 0.972 \\
\hline Other non-psychotic disorder & $6(60)$ & $4(40)$ & 0.112 & 1 & 0.738 \\
\hline \multicolumn{6}{|l|}{ Treatment, $n(\%)$} \\
\hline Antipsychotic & $46(54.1)$ & $39(45.9)$ & 0.178 & 1 & 0.673 \\
\hline Typical antipsychotic monotherapy & $3(50)$ & $3(50)$ & 0.064 & 1 & 0.800 \\
\hline Atypical antipsychotic & $43(54.4)$ & $36(45.6)$ & 0.049 & 1 & 0.08 \\
\hline Antipsychotic polypharmacy & $16(50)$ & $16(50)$ & 0.475 & 1 & 0.491 \\
\hline High-dose antipsychotic & $5(31.3)$ & $11(68.8)$ & 4.341 & 1 & 0.043 \\
\hline Clozapine & $7(77.8)$ & $2(22.2)$ & 2.073 & 1 & 0.150 \\
\hline Olanzapine & $21(61.8)$ & $13(38.2)$ & 0.952 & 1 & 0.329 \\
\hline Smoking, yes & $25(58.1)$ & $18(41.8)$ & 1.211 & 2 & 0.546 \\
\hline Exercise $>3 \times 20 \mathrm{~min} /$ week & $19(45.2)$ & $23(54.8)$ & 2.760 & 1 & 0.071 \\
\hline Family history of CVD & $21(52.5)$ & $19(47.5)$ & 0.168 & 1 & 0.682 \\
\hline Family history of diabetes & $13(61.9)$ & $8(38.1)$ & 0.512 & 1 & 0.47 \\
\hline
\end{tabular}

CVD, cardiovascular disease.

a. Independent sample $t$-test.

monotherapy; $16 \%$ were on a high-dose antipsychotic and $32 \%$ were taking more than one antipsychotic.

Fifty-five patients (55\%) met the IDF criteria for metabolic syndrome. The clinical characteristics of patients with and without the metabolic syndrome are presented in Table 2. Relatively more women than men met the diagnostic criteria for metabolic syndrome, but this did not reach statistical significance $(P=0.089)$. There was no difference in the prevalence of metabolic syndrome by psychiatric diagnosis. The means of most metabolic parameters were significantly elevated in those patients fulfilling criteria for the metabolic syndrome. However, the mean total cholesterol levels were significantly lower in the group of patients with the metabolic syndrome than in those without the metabolic syndrome, presumably because $40 \%$ of individuals with the syndrome were on statin therapy, compared with $13 \%$ without metabolic syndrome on statin therapy.
Overall, 54\% of patients on atypical antipsychotics, $50 \%$ of those on antipsychotic polypharmacy and $31 \%$ of those on high-dose antipsychotic medication met the criteria for metabolic syndrome; however, there was no statistically significant association between each of these variables and the metabolic syndrome. Indeed, those taking high-dose antipsychotic medication were actually less likely to have metabolic syndrome. Most of the patients taking clozapine $(78 \%)$ or olanzapine (62\%) met the criteria for metabolic syndrome, although this was not a statistically significant association. Eleven per cent of individuals were on antidepressant monotherapy and $64 \%(n=7)$ of those patients met the criteria for the metabolic syndrome.

The proportion of the sample, divided by gender, which met each of the individual criteria for metabolic syndrome is displayed in Table 3: $88 \%$ of individuals met the criteria for central obesity, $41 \%$ for hypertension and $43 \%$ for hypertriglyceridaemia. There were $32 \%$ of individuals with

\begin{tabular}{|c|c|c|c|c|c|c|}
\hline & \multirow[b]{2}{*}{ Total } & \multicolumn{2}{|c|}{$n(\%)$} & \multirow[b]{2}{*}{$\chi^{2}$} & \multirow[b]{2}{*}{ d.f. } & \multirow[b]{2}{*}{$P^{\mathrm{a}}$} \\
\hline & & Male $(n=44)$ & Female $(n=56)$ & & & \\
\hline Metabolic syndrome & 55 & $20(43.5)$ & $35(62.5)$ & 2.893 & 1 & 0.089 \\
\hline Central obesity & 88 & $36(81.8)$ & $52(92.8)$ & 2.843 & 1 & 0.092 \\
\hline Hypertension & 41 & $16(36.4)$ & $25(44.6)$ & 0.833 & 1 & 0.362 \\
\hline Hypertriglyceridaemia & 43 & $20(45.5)$ & $23(41.1)$ & 0.216 & 1 & 0.642 \\
\hline Low HDL & 29 & $11(25)$ & $18(32.1)$ & 0.506 & 1 & 0.477 \\
\hline Glucose dysregulation & 32 & $14(21.8)$ & $18(32.1)$ & 0.070 & 1 & 0.936 \\
\hline
\end{tabular}

$\mathrm{HDL}$, high-density lipoprotein.

a. $\chi^{2}$-test between males and females. 




Fig. 1 Rates of metabolic abnormalities of patients who were not monitored for metabolic risk factors.

HbA1c, glycated haemoglobin; HyperTG, hypertriglyceridaemia; HDL, high-density lipoprotein; LDL, low-density lipoprotein.

evidence of glucose dysregulation (impaired glucose tolerance or undiagnosed diabetes mellitus, or diagnosis of diabetes mellitus, or HbAlc $>6 \%){ }^{20}$ This is in contrast to the figure of $14 \%$ for previously diagnosed diabetes mellitus in our study population. Whereas $28 \%$ of our study population were on treatment for hypercholesterolaemia and $33 \%$ were on anti-hypertensive medication, $47 \%$ of patients with metabolic syndrome were being treated with anti-hypertensive medication.

A substantial proportion (44\%) of the individuals in this study had not been monitored for metabolic parameters in the previous year and this included $35 \%$ of the total population who were taking antipsychotic medication (Fig. 1). This cohort of unscreened individuals had a prevalence rate of $43 \%$ for the metabolic syndrome, with $30 \%$ identified with glucose dysregulation, including two new cases of diabetes mellitus. The rate of dyslipidaemia was greater than $50 \%$ in these individuals.

\section{Discussion}

There was a high prevalence rate of metabolic syndrome in this typical cohort of individuals with chronic enduring mental illness attending day centres within the catchment area service. Over half of these individuals (55\%) met the criteria for metabolic syndrome, which is over twice the prevalence in the general Irish population. ${ }^{21}$ This prevalence rate is similar to a previous study which examined patients on clozapine therapy and identified $46 \%$ prevalence rate for the metabolic syndrome. ${ }^{22}$

There was a similar prevalence of the metabolic syndrome in individuals with a history of non-psychotic disorders and those with a history of psychotic disorders. This study sample is representative of a typical cohort of patients with chronic enduring mental illness and there is a significantly elevated prevalence of the metabolic syndrome across a broad range of psychiatric disorders. This pattern of metabolic syndrome existing across psychiatric disorders has been reported in other studies. ${ }^{9,23,24}$ This study provides further evidence that chronic enduring mental illness in general should be considered a risk factor for metabolic syndrome. A similar prevalence rate of metabolic syndrome (using the IDF criteria) of $51 \%$ in patients with schizophrenia was reported elsewhere ${ }^{25}$ and has been replicated in other studies such as the finding of a prevalence rate of $49 \%$ in individuals with a duration of schizophrenia of more than 20 years. ${ }^{26}$ The high rate of metabolic syndrome in our study regardless of diagnostic group or the use of antipsychotic medication indicates that it is chronic mental illness per se which is associated with metabolic syndrome rather than a psychotic diagnosis or the use of atypical antipsychotic medication. This may be due to the poor self-care associated with this patient cohort, including unhealthy diet and sedentary lifestyle.

Our study demonstrated similar prevalence rates of the metabolic syndrome in individuals treated with antipsychotics (54\%) and those not on antipsychotic medications (46\%). This is similar to other studies which showed prevalence rates of $48 \%$ for the metabolic syndrome in individuals treated with antipsychotics. ${ }^{14}$

Strengths of the study are the screening of a cohort attending day centres in a catchment area service and thus representative of patients with chronic enduring mental illness in the region, rather than confined to a particular diagnosis or type of medication.

Limitations of the study include the cross-sectional design and the lack of a non-medicated control group or longitudinal assessment. This study did not assess the severity of symptoms, which may have been a contributing factor to the presence of the metabolic syndrome. Other confounding factors which may act as independent risk factors for metabolic syndrome (e.g. poor diet) were not assessed in this study population.

Despite the high prevalence of cardiovascular risk factors in patients with chronic enduring mental illness, such patients are likely to suffer from a fragmented and inconsistent approach to their physical healthcare. It has been identified that people with long-term mental illness tend to seek medical attention only in crisis situations and have limited access to general healthcare, with less opportunity for cardiovascular disease risk screening and prevention. ${ }^{27}$ This, allied to the high rates of metabolic 
syndrome in this cohort, highlights the need for improved integration in the screening for and management of physical risk factors. It is particularly important to improve the collaboration between primary and secondary care in the clinical care of these individuals. A recent study by our group has indicated that the majority of GPs were willing to take over the medical management of metabolic dysregulation emerging from antipsychotic prescribing in secondary care. ${ }^{28}$ Given the high prevalence of metabolic risk factors in individuals with chronic enduring mental illness regularly attending mental health services, it is important that mental health services take advantage of the opportunity to improve their care by implementing protocols for the screening, pharmacological (including alteration of psychotropic medication) and non-pharmacological management of these risk factors and referral to primary or secondary care as required. As a minimum, individuals who are treated with antipsychotics should be systematically screened according to guidelines. ${ }^{29}$ The results of this study which showed high prevalence rates for metabolic syndrome across a range of psychotic and affective disorders indicates that it could be beneficial to extend such guidelines to the wider cohort of patients with chronic enduring mental illness. Twenty-five patients with metabolic syndrome in our cohort smoked (45.5\%). Effective smoking cessation programmes would be an important intervention in reducing this particular cardiovascular risk factor.

A recent systematic review demonstrated that there is a paucity of research on the impact of general physical health advice on the outcomes of physical health awareness or physical health behaviour in people with serious mental illness. ${ }^{30}$ There is a general consensus that physical activity has a mild to moderately favourable effect on many metabolic and cardiovascular risk factors that constitute or are related to metabolic syndrome. ${ }^{31}$ This is supported by our study which demonstrated that $55 \%$ of individuals who exercised for more than $20 \mathrm{~min}$ at a time on more than three occasions in a week did not meet the criteria for metabolic syndrome. Physical health and diet educational programmes as non-pharmacological interventions for weight gain and other metabolic abnormalities should be implemented in the community setting. ${ }^{32,33}$ This will involve initiation of programmes which can be adapted to the individuals' personal preferences and their personal attitudes towards physical exercise and dietary restrictions.

The high prevalence of the metabolic syndrome in those with a history of chronic enduring mental illness indicates that this population would benefit from regular screening for and the intensive management of cardiometabolic risk factors to reduce their morbidity and mortality secondary to cardiovascular disease.

\section{Funding}

Research Support Fund, National University of Ireland, Galway.

\section{Acknowledgements}

We thank the staff and patients of the Ceim Eile and Danesfield psychiatric day centres in Galway city and the staff and patients of the Elm Tree Centre,
Clifden, Co. Galway for their assistance in the completion of this study. We thank Mr Ben Kanagaratnam for his advice on the statistical analyses.

\section{About the authors}

Anna Gubbins is a 4th-year medical student at National University of Ireland, Galway. John Lally (MB, MRCPsych, PG Dip Clinical Education) is a Clinical Lecturer in Psychiatry and Colm McDonald (PhD MRCPsych) is Professor of Psychiatry at National University of Ireland, Galway; both work at West Galway Mental Health Services, Health Service Executive West.

\section{References}

1 Brown S, Barraclough B, Inskip H. Causes of the excess mortality of schizophrenia. Br J Psychiatry 2000; 177: 212-7.

2 Angst F, Stassen $\mathrm{HH}$, Clayton PJ, Angst J. Mortality of patients with mood disorders: follow-up over 34-38 years. J Affect Disord 2002; 68: 167-81.

3 Sukanta S, Chant D, McGrath J. A systematic review of mortality in schizophrenia is the differential mortality gap worsening over time? Arch Gen Psychiatry 2007; 64: 1123-31.

4 Brown S. Excess mortality of schizophrenia. A meta-analysis. $\mathrm{Br}$ Psychiatry 1997; 171: 502-8.

5 Hoang U, Stewart R, Goldacre MJ. Mortality after hospital discharge for people with schizophrenia or bipolar disorder: retrospective study of linked English hospital episode statistics, 1999-2006. BMJ 2011; 343: d5422.

6 De Hert M, Dekker JM, Wood D, Kahl KG, Holt RIG, Möller HJ. Cardiovascular disease and diabetes in people with severe mental illness position statement from the European Psychiatric Association (EPA), supported by the European Association for the Study of Diabetes (EASD) and the European Society of Cardiology (ESC). Eur Psychiatry 2009; 24: 412-24.

7 Alberti KGMM, Zimmet P, Shaw J. The metabolic syndrome - a new worldwide definition. Lancet 2005; 366: 1059-62.

8 Khatana SAM, Kane J, Taveira TH, Bauer MS, Wen-Chih W. Monitoring and prevalence rates of metabolic syndrome in military veterans with serious mental illness. PLoS One 2011; 6: 1-8.

9 Daumit G, Goff D, Meyer J, Davis V, Nasrallah H, McEvoy J, et al. Antipsychotic effects on estimated 10-year coronary heart disease risk in the CATIE schizophrenia study. Schizophr Res 2008; 105: 175-87.

10 Umbricht DSG, Pollack S, Kane JM. Clozapine and weight gain. J Clin Psychiatry 1994; 55: 157-60.

11 Allison DB, Mentore JL, Heo M, Chandler LP, Cappelleri JC, Infante MC et al. Antipsychotic-induced weight gain: a comprehensive research synthesis. Am J Psychiatry 1999; 156: 1686-96.

12 Akhtar S, Kelly C, Gallagher A, Petrie JR. Newer antipsychotic agents, carbohydrate metabolism and cardiovascular risk. Br J Diab Vasc Dis 2004; 4: 303-9.

13 Newcomer JW. Second-generation (atypical) antipsychotics and metabolic effects: A comprehensive literature review. CNS Drugs 2005; 19: 1-93.

14 Krane-Gartiser K, Breum L, Glümrr C, Linneberg A, Madsen M, Køster A, et al. Prevalence of the metabolic syndrome in Danish psychiatric outpatients treated with antipsychotics. Nord J Psychiatry 2011; 65: 345-52.

15 Correll CU, Druss BG, Lombardo I, O'Gorman C, Harnett JP, Sanders KN et al. Findings of a U.S. national cardiometabolic screening program among 10,084 psychiatric outpatients. Psychiatr Serv 2010; 61: 892-8.

16 Gumber R, Abbas M, Minajagi M. Monitoring the metabolic side-effects of atypical antipsychotics. Psychiatrist 2010; 34: 390-5.

17 World Health Organization. International Statistical Classification of Diseases and Related Health Problems (10th revision) (ICD-10). WHO, 1992.

18 British Medical Association, Royal Pharmaceutical Society of Great Britain. British National Formulary. BMJ Group, Pharmaceutical Press. 
19 Yorston G, Pinney A. Chlorpromazine equivalents and percentage of British National Formulary maximum recommended dose in patients receiving high-dose antipsychotics. Psychiatr Bull 2000; 24: 130-2.

20 Nathan DM, Balkau B, Bonora E, Borch-Johnsen K, Buse JB, Colagiuri S, et al. International expert committee report on the role of the $\mathrm{A} 1 \mathrm{C}$ assay in the diagnosis of diabetes. CPD Bull Clin Biochem 2010; 10: 25-33.

21 Villegas R, Perry IJ, Creagh D, Hinchion R, O'Halloran D. Prevalence of the metabolic syndrome in middle-aged men and women. Diab Care 2003; 26: 3198-9.

22 Ahmed M, Hussain I, O'Brien SM, Dineen B, Griffin D, McDonald C. Prevalence and associations of the metabolic syndrome among patients prescribed clozapine. Ir J Med Sci 2008; 177: 205-10.

23 Mackin P, Bishop D, Watkinson H, Gallagher P, Ferrier IN. Metabolic disease and cardiovascular risk in people treated with antipsychotics in the community. Br J Psychiatry 2007; 191: 23-9.

24 Vinberg M, Madsen M, Breum L, Kessing LV, Fink-Jensen A. Metabolic syndrome in a cohort of affectively ill patients, a naturalistic study. Nord J Psychiatry 2012; 66: 142-5.

25 John AP, Koloth R, Dragovic M, Lim SCB. Prevalence of metabolic syndrome among Australians with severe mental illness. Med J Australia 2009: 190: 176-9.

26 De Hert M, van Winkel R, Van Eyck D, Hanssens L, Wampers M, Scheen $A$, et al. Prevalence of diabetes, metabolic syndrome and metabolic abnormalities in schizophrenia over the course of the illness: a crosssectional study. Clin Pract Epidemiol Ment Health 2006; 2: 14.

27 Kendrick T. Management of people with schizophrenia in primary care. Adv Psychiatr Treat 1998; 4: 46-52.

28 Bainbridge E, Gallagher A, McDonald G, McDonald C, Ahmed M General practitioners' attitudes on who should manage metabolic dysregulations associated with antipsychotics. Psychiatrist 2011; 35: 213-5.

29 De Hert M, Vancampfort D, Correll CU, Mercken V, Peuskens J, Sweers $K$, et al. Guidelines for screening and monitoring of cardiometabolic risk in schizophrenia: systematic evaluation. $\mathrm{Br} J$ Psychiatry 2011; 199: 99-105.

30 Tosh G, Clifton A, Bachner M. General physical health advice for people with serious mental illness. Cochrane Database Syst Rev 2011; 2 CD008567.

31 De Hert M, Schreurs V, Vancampfort D, Van Winkel R. Metabolic syndrome in people with schizophrenia: a review. World Psychiatry 2009; 8: 15-22.

32 Holt RIG, Abdelrahman T, Hirsch M, Dhesi Z, George T, Blincoe T, et al. The prevalence of undiagnosed metabolic abnormalities in people with serious mental illness. J Psychopharmacol 2010; 24: 867-73.

33 Evans S, Newton R, Higgins S. Nutritional intervention to prevent weight gain in patients commenced on olanzapine: a randomized controlled trial. Aust N Z J Psychiatry 2005; 39: 479-86.

\title{
Crisis plans in a home treatment team before and after a quality improvement programme
}

\author{
Emily Tan, ${ }^{1}$ Andrew Higginbotham, Lorette McQueen, ${ }^{1}$ Kamaldeep Bhui ${ }^{1,2}$
}

The Psychiatrist (2012), 36, 331-334, doi: 10.1192/pb.bp.111.035956

${ }^{1}$ East London Foundation Trust;

${ }^{2}$ Wolfson Institute of Preventive

Medicine, Barts \& The London School

of Medicine and Dentistry, Queen

Mary University of London

Correspondence to Kamaldeep Bhui

(k.s.bhui@qmul.ac.uk)

First received 7 Jul 2011, final revision 13 Feb 2012, accepted 22 Mar 2012

\begin{abstract}
Aims and method To measure the take-up of formal crisis plans in a specialist home treatment team (HTT) serving an ethnically diverse urban population; and to implement a quality improvement programme and measure its impact on crisis plan completion. Two audits were completed of patients before and after the implementation of a quality improvement programme. Descriptive data are shown by ethnic group, by gender, and for people with multiple hospital admissions.
\end{abstract}

Results At baseline, $16.7 \%$ of patients at high risk of admission had an HTT crisis plan, compared with $26.7 \%$ of a comparison group. Only $23.1 \%$ of the crisis plans for patients with a history of frequent admission mentioned the prevention of future admissions. Crisis plan completion improved following the quality improvement programme, when $80.0 \%$ of discharges had an HTT crisis plan; of these, $73.0 \%$ mentioned admission prevention. In the follow-up audit, $22.7 \%$ of patients in the multiple admission group had been readmitted to hospital at least once. Crisis plan completion did not appear to differ by ethnic group or gender and did not appear to be related to hospital readmission.

Clinical implications Crisis plan completion improved with simple practical methods, but completion was unrelated to gender, ethnicity or later readmission.

Declaration of interest None.
Collaborative and person-centred crisis plans are part of mental health policy in the UK, but little work has investigated take-up of crisis plans or equity in their use by crisis resolution and home treatment teams (CRHTs).
Crisis plans may be a useful tool in the prevention of future hospital admissions, and they are especially relevant for CRHTs that perform a central gatekeeping role for people at risk of admission to hospital. Previous evaluations of 